\title{
Heat Transfer Experiments of Granular Medium in a Rotating Drum with Various Wall Roughness
}

\author{
Li-Tsung Sheng, Shu-San Hsiau, Chia-Hsien Chang \\ Department of Mechanical Engineering, National Central University \\ 300 Jhongda Road, Jhongli 32001, Taiwan \\ li.tsung.sheng@gamil.com; sshsiau@cc.ncu.edu.tw; tysh1016@gmail.com
}

\section{Extended Abstract}

Rotating drums/kilns are processing facilities used in industry for thermal treatment of various granular materials. Some applications are sintering of cement, calcinations of limestone and coke, drying of food products, and incineration of waste. The main purpose of the thermal treatment of the rotating drums is to raise the temperature of the material to the target temperature so that a desired chemical or physical process is taken place. Mostly, rotating drums heat the material directly from the sidewalls of the drums. Due to the importance of heat transfer of the bulk material in the rotating drum in industrial applications, this issue has already been investigated by many literatures [1-3]. The heat transfer in rotating drums strongly depends on the combined transport of mass and heat within the granular material. Thus, there will exist an obvious different heat transfer behaviour if the rotating drum has different sidewall roughness. This is because that the dynamics of granular flow in the drum may be changed by the different sidewall condition.

In this study, a quasi-two-dimensional rotating heated system is used to investigate the heat transfer behaviour. A circular heater is mounted on the outside of the sidewall (made by steel) of the drum to heat the wall. Several rotating speeds also are adopted in this study. The sidewall is heated with $140 \mathrm{~W}$ and the temperature of the sidewall continuously increases from $298 \mathrm{~K}$ to $333 \mathrm{~K}$. The glass beads with a nominal diameter of $2 \mathrm{~mm}$ are filled to $30 \%$ filling level of the drum. The inside of the sidewall is randomly adhered with glass beads to produce the roughness of wall. An infrared camera is used to measure the temperature fields of the granular beds. Fig. 1 (left) shows one of the image of infrared thermography measurement in this study and some details of rotating drum also are indicated in the Fig.1. The heat transfer coefficients of the rotating heating system are calculated to investigate the effect of sidewall roughness on the heat transfer behaviour of this system. Another rotating system with the same size as the rotating heating system and without the heating structure, but the front wall is made from transparent Plexiglas, to allow experimental images to be captured by the high speed camera. The experimental images are analysed to observe the dynamics of the glass beads in the rotating drum, see the Fig.1 (right). Finally, the results of the dynamics of granular bed can be reasonable to explain the results of the heat transfer behaviour of the rotating heated system with different sidewall roughness.

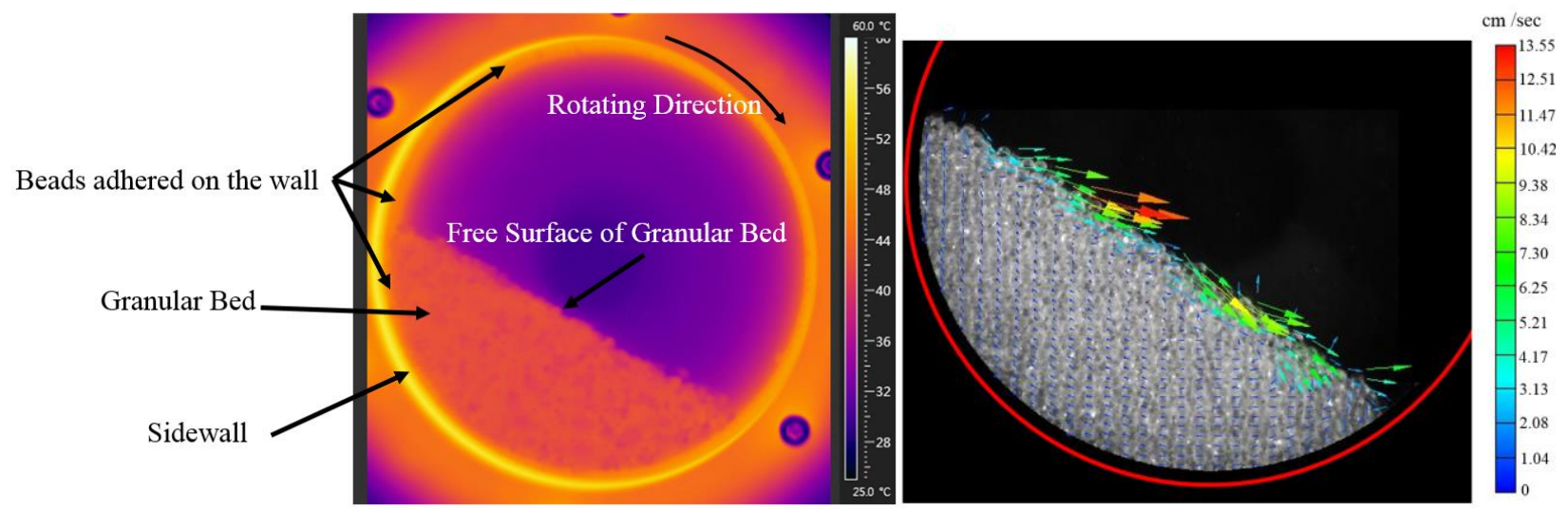

Fig. 1: (Left) The image of the temperature filed from the infrared thermography measurement. (Right) The velocity field of the glass beads in the rotating drum by PIV measurement. 


\section{References}

[1] B. Chaudhuri, F. J. Muzzio, M. S.Tomassone, "Experimentally validated computations of heat transfer in granular materials in rotary calciners," Powder Technol. vol. 198, pp. 6-15, 2010.

[2] H. Komossa, S. Wirtz, V. Scherer, F. Herz, E. Specht, "Heat transfer in indirect heated rotary drums filled with monodisperse spheres: Comparison of experiments with DEM simulations," Powder Technol. vol. 286, pp. 722-731, 2015.

[3] A. I. Nafsun, F. Herz, E. Specht, V. Scherer, and S. Wirtz, "Heat transfer experiments in a rotary drum for a variety of granular materials," Exp. Heat Transf., vol. 29, pp. 1-16, 2016. 